\title{
Revalidation of CoaguChek XS Plus System for INR Monitoring in Taiwanese Patients: Effects of Clinical and Genetic Factors
}

\author{
Chao-Hua Fu ${ }^{1}$, Wei-Ting Chen ${ }^{2}$, Pi-Yueh Chang ${ }^{1,3}$, Ming-Ta Michael Lee ${ }^{4,5}$, Ming-Shien Wen ${ }^{1,6}$
}

Background: In this study, we evaluated the performance of a point-of-care device, the CoaguChek XS Plus system, in the determination of prothrombin time and international normalized ratio (INR) based on ISO17593: 2007 criteria in Taiwanese patients. The underlying clinical and genetic factors were also investigated.

Methods: Fifty patients receiving warfarin therapy were enrolled in this study. The accuracy of the CoaguChek XS Plus system was evaluated with linear regression analysis and bias plot by comparing with the data measured using Sysmex CA-1500. The clinical and genetic factors that may have caused a bias of $\geq 0.5$ INR were evaluated with Fisher's exact test.

Results: $\quad$ From the 50 patients, 93 INR values were collected by each method. Linear regression analysis indicated a high correlation with $r=0.96$, a slope of 1.05 , and an intercept of -0.14 . Eight patients showed an INR bias $\geq 0.5$ between the two methods. Only aspartate aminotransferase (AST) $>34 \mathrm{U} / \mathrm{L}(3 / 8,37.5 \%$ vs. $3 / 42,7.1 \% ; p=0.044)$ and alanine aminotransferase (ALT) $>36 \mathrm{U} / \mathrm{L}(3 / 8,37.5 \%$ vs. $3 / 42$, $7.1 \% ; p=0.044$ ) were significantly different from each other. No differences were observed for hypoalbuminemia, elevated creatinine, anemia, and the polymorphisms of VKORC1 and CYP2C9.

Conclusions: The CoaguChek XS Plus system presented results that were comparable with those obtained using laboratory CA-1500 method. Both methods fell within INR in the range of 2-4.5 defined by ISO17593:2007 and the clinically recognized therapeutic INR range of 2-3.5. Elevated AST and ALT levels might have interfered with the INR results.

(Biomed J 2014;37:380-385)

\section{At a Glance Commentary}

Scientific background of the subject

CoaguChek XS plus system was released in October 2005, what served as a fast and convenient device for INR monitoring. Patients receiving warfarin needs frequent INR monitor to avoid drug adverse reaction which may cause death. In this study, CoaguChek XS plus system was validated compared to laboratory CA-1500. Although CoaguChek XS system had been validated worldwide, Taiwan patients had not been assessed in clinical practice. This is the first CoaguChek XS performance study in Taiwan. The potential genetic factors and clinical factors that may interfere test are assessed.

\section{What this study adds to the field}

This study find that CoaguChek XS plus system reach about $90 \%$ clinical agreement compared to laboratory CA-1500 and elevated AST and ALT level have effect on CoaguChek XS system analysis.

\section{Key words: CoaguChek XS system, interference, ISO17593:2007, point-of-care, warfarin}

C.H. Fu and W.T. Chen Contributed equally to this article.

From the ${ }^{1}$ Department of Laboratory Medicine, Chang Gung Memorial Hospital at Linkou, Chang Gung University College of Medicine, Taoyuan, Taiwan; ${ }^{2}$ Department of Medicine, Second Section of Cardiology, Chang Gung Memorial Hospital at Linkou, Chang Gung University College of Medicine, Taoyuan, Taiwan; ${ }^{3}$ Department of Medical Biotechnology and Laboratory Science, Chang Gung University, Taoyuan, Taiwan; ${ }^{4}$ Institute of Information Science, Academic Sinica, Taipei, Taiwan; ${ }^{5}$ Laboratory for Genotyping Development, Center for Genomic Medicine, RIKEN, Yokohama Institute, Yokohama, Japan; ${ }^{6}$ School of Medicine, Chang Gung University, Taoyuan, Taiwan Received: Jul. 23, 2013; Accepted: Jan. 13, 2014

Correspondence to: Dr. Pi-Yueh Chang, Department of Laboratory Medicine, Chang Gung Memorial Hospital at Linkou. 5, Fusing St., Gueishan, Taoyuan 333, Taiwan (ROC). Tel: 886-3-3281200 ext. 5150; Fax: 886-3-3282997; E-mail: changpy@ adm.cgmh.org.tw Correspondence to: Dr. Ming-Shien Wen, Department of Laboratory Medicine, Chang Gung Memorial Hospital at Linkou. 5, Fusing St., Gueishan, Taoyuan 333, Taiwan (ROC). Tel: 886-3-3281200 ext. 5150; Fax: 886-3-3282997; E-mail: wenms123@gmail.com

DOI: $10.4103 / 2319-4170.132904$ 
W arfarin, a widely prescribed oral anticoagulant, prevents thromboembolism in patients with deep vein thrombosis, atrial fibrillation, or prosthetic heart valve replacements. However, warfarin has a narrow therapeutic index, making it difficult for physicians to maintain the dose in a therapeutic range. To prevent warfarin-associated adverse reactions, it is advised that for patients receiving warfarin, the international normalized ratio (INR) is maintained within the range of 2.0-3.0 for atrial fibrillation and other thromboembolic disorders and within the range of 2.5-3.5 for mechanical heart valve patients. ${ }^{[1,2]}$ Patients with an INR exceeding 4.0 showed an increased risk of hemorrhaging, and patients were more likely to suffer from embolism if their INR value was lower than the therapeutic range. Most warfarin adverse reactions occur when treatment is initiated. Therefore, the INR must be monitored frequently after initiation of warfarin treatment. However, for outpatients receiving warfarin in Taiwan, frequent INR monitoring during the first 2 weeks is difficult. There are two reasons for this. First, outpatients undergo the INR test only when prescribed to do so by a physician; and second, frequent withdrawal of blood within a short time period adds to patients' discomfort levels. A non-invasive, quick INR test is essential to improve patient compliance with frequent INR monitoring.

The CoaguChek XS Plus system, a portable coagulation monitoring system, provides fast and effective point-of-care monitoring, with a measurement range of 0.8-8.0 INR ${ }^{[3]}$ Although the performance of the CoaguChek XS Plus system has been evaluated worldwide, the device has not been validated in Taiwan. To improve patient compliance, the CoaguChek XS Plus system is aimed at patients receiving warfarin in Taiwan. In this study, we evaluated the CoaguChek XS Plus system against the Sysmex CA-1500 system, using CA-1500-Thromborel S reagent, based on the ISO 17593:2007 standard criteria. ${ }^{[4]}$ The standard stipulates the requirements for in vitro monitoring systems for oral anticoagulant therapy for both self-testing medical devices and clinical laboratory settings. Additionally, we evaluated the clinical and genetic factors that might cause a bias of $\geq 0.5$ INR between the two methods.

Since CoaguChek XS system was released in October 2005, the performance of the device was evaluated ${ }^{[3,5,6]}$ As a portable device, the practice in self-management was studied ${ }^{[7-9]}$ And the convenience of its small sample need, the practice in children were accessed. ${ }^{[10,11]}$ Nowadays, the device was studied in different medical conditions, such as patients with ventricular assist devices or patients receiving telavancin. ${ }^{[11,12]}$ Although CoaguChek XS system had been validated worldwide, Taiwan patients had not been assessed in clinical practice. This is the first CoaguChek XS performance study in Taiwan. Moreover, the interference of clinical and genetic factors were discussed beyond other studies.

\section{METHODS}

\section{Study subjects and point-of-care procedure for INR measurements}

For this study, we enrolled 50 patients who received warfarin therapy from 25 June 2010 to 3 June 2011. The initial study design involved obtaining paired samples in two separate visits after each patient had received their initial warfarin prescription. However, seven patients did not participate in the second sample collection. Thus, 93 INR values for each method were collected. Trained technicians collected the capillary blood (by finger prick) for the CoaguChek XS Plus system immediately before venipuncturing for the central laboratory CA-1500 analysis of prothrombin time (PT), INR, and other blood tests. Biochemical data were collected during the first visit. This study protocol was approved by the Institutional Review Board of Chang Gung Memorial Hospital.

The CoaguChek XS Plus system (Roche Diagnostics, Mannheim, Germany) consisted of the CoaguChek XS monitor and CoaguChek XS PT test strips. The device used a human recombinant thromboplastin with an International Sensitivity Index (ISI) value of 1.0 to activate the coagulation cascade in blood. The production of thrombin cleaves a peptide substrate on the test strip to generate an electrochemical signal. In this study, the system was calibrated according to the manufacturer's manual, the information of which was stored in a lot-specific microchip code included in every vial of the test strips. The results were presented as the PT in INR, \%Quick, or seconds. Only the INR values were used for analysis.

\section{Laboratory procedure}

For the laboratory tests, venous blood samples were drawn by standard procedures into $3.2 \%$ sodium citrate tubes, serum-separating tubes, and dipotassium ethylenediaminetetraacetic acid (K2-EDTA) tubes $(4.08 \mathrm{mM}$, final concentration). For the PT test, the centrifuged plasma was analyzed with the Sysmex CA-1500 (Sysmex Corporation, Kobe, Japan). The thromboplastin reagent used for the laboratory test was Thromborel S (Siemens Healthcare Diagnostics, Marburg, Germany), with an ISI of 0.95 . Thromborel S reagent is lyophilized human placental thromboplastin. For the aspartate aminotransferase (AST), alanine aminotransferase (ALT), albumin, and creatinine tests, the centrifuged sera were analyzed with a Hitachi 7600 
analyzer (Hitachi Inc., Tokyo, Japan). A complete blood count (CBC) test was done with Sysmex XE-5000 (Sysmex Corporation, Kobe, Japan). The genotyping method for $\mathrm{CY}$ P2C9 (rs1057910, CYP2C9*3) and VKORC1 (rs9923231, VKORC1-1639 G > A) has been described previously. ${ }^{[13]}$

\section{Performance validation}

\section{Precision of the CoaguChek XS Plus system}

The precision of the CoaguChek XS Plus system was evaluated using the Roche CoaguChek XS PT control with an INR value of approximately 1.8. Within-run precision was tested eight times in one run, and between-day precision was tested for 8 days. Finally, the mean INR, standard deviation (SD), and coefficient of variation (CV) were calculated.

\section{Accuracy of the CoaguChek XS Plus system}

The ISO17593:2007 standard "Clinical laboratory testing and in vitro medical devices - Requirements for in vitro monitoring systems for self-testing of oral anticoagulant therapy" provided the criteria for evaluating the performance of in vitro monitoring systems for self-testing of oral anticoagulant therapy, such as the CoaguChek XS Plus system.

The three point-of-care testing (POCT) performance-acceptance criteria of the ISO17593:2007 standards are as follows:

- The ISO standard stipulates a slope between 0.95 and 1.05 and an intercept between $\pm 0.1 \mathrm{INR}$, in linear regression analysis

- The mean bias in the INR range of 2.0-4.5 should be within \pm 0.3 INR. (Bias = CoaguChek XS Plus INR laboratory CA-1500 INR)

- More than $90 \%$ of the bias in the INR range below 2 INR must be within \pm 0.5 INR and in the INR range of 2.0 to 4.5 the bias must be within $\pm 30 \%$.

Because the therapeutic range claimed by ISO 17593:2007 is 2.0 to 4.5 , we categorized the INR values as $<2.0,2.0-4.5$, and $>4.5$, to meet the requirements of the ISO standard. The INR values of both methods were compared using a paired $t$ test ( $p<0.05$ was considered significant).

\section{Clinical agreement}

Clinical agreement was defined based on whether a bias existed between the results of the two methods for various warfarin dosages. Based on the standard therapeutic range of 2.0-3.0 for atrial fibrillation and 2.5-3.5 for patients with mechanical heart valves, we categorized the INR values as $<2.0$, between 2.0 and 3.5, and $>3.5$ to fit most of the clinical therapeutic range. The percentage of INR results reported by the CoaguChek XS Plus system and the laboratory CA-1500 method that fell within the same dosage categories was calculated.

\section{Interferences of clinical and genetic factors}

We found that several patients showed a high discrepancy in results between the CoaguChek XS Plus system and the laboratory CA- 1500 method, with an INR bias $\geq 0.5$. Depending on the method used, a different clinical decision for warfarin dosage may have been made by a physician, which may have caused an adverse outcome. Clinical and genetic factors were examined to determine whether any of these factors were able to cause differences in the INR ( $\geq 0.5$ INR) between the CoaguChek XS Plus and laboratory CA-1500 methods. Clinical factors analyzed included the patient's demography (age, gender), biochemical data (AST, ALT, creatinine, albumin and hematocrit levels), and genetic data of CYP2C9 (rs1057910, CYP2C9*3) and VKORC1 (rs9923231, VKORC1-1639 G > A). We used Fisher's exact test to examine the significance of the results.

\section{RESULTS}

Of the 50 patients enrolled for this study, $62 \%$ were males and $38 \%$ were females. Fifty percent of the group exhibited atrial fibrillation, $32 \%$ showed deep vein thrombosis, and $18 \%$ showed other indications. The median age was 68 years. The 93 INR values collected ranged from 1.2 to 7.7 when measured with the CoaguChek XS Plus system, and from 1.2 to 6.5 when measured with the laboratory CA-1500 method. The mean INR and SD for each subgroup are shown in Table 1.

Table 1: Accuracy evaluation of INR results classified by ISO 17593:2007 standard

\begin{tabular}{|c|c|c|c|}
\hline & $\begin{array}{c}\text { Laboratory } \\
\text { CA-1500 }\end{array}$ & $\begin{array}{l}\text { CoaguChek XS } \\
\text { Plus system }\end{array}$ & $p$ value* \\
\hline Entire INR range, $n(\%)$ & $93(100 \%)$ & & \\
\hline Mean INR (SD) & $2.54(1.14)$ & $2.52(1.24)$ & 0.759 \\
\hline Mean INR bias (range) & & -0.01 ( -0.5 to 2.2$)$ & \\
\hline Bias 0.5 INR (within 30\%) & & $96 \%(98 \%)$ & \\
\hline $\mathrm{INR}<2.0, n(\%)$ & $31(33 \%)$ & & \\
\hline Mean INR (SD) & $1.61(0.22)$ & $1.61(0.22)$ & 0.913 \\
\hline Mean bias (range) & & $0(-0.2$ to 0.6$)$ & \\
\hline Bias 0.5 INR & & $97 \%$ & \\
\hline INR $2.0-4.5, n(\%)$ & $54(58 \%)$ & & \\
\hline Mean INR (SD) & $2.63(0.6)$ & $2.57(0.56)$ & 0.125 \\
\hline Mean INR bias (range) & & $-0.06(-0.5$ to 0.8$)$ & \\
\hline Bias within $30 \%$ & & $100 \%$ & \\
\hline $\mathrm{INR}>4.5, n(\%)$ & $8(9 \%)$ & & \\
\hline Mean INR (SD) & $5.51(0.72)$ & $5.78(1.27)$ & 0.424 \\
\hline Mean INR bias (range) & & $0.26(-0.3$ to 2.2$)$ & \\
\hline Bias within $30 \%$ & & $88 \%$ & \\
\hline
\end{tabular}

Abbreviations: INR: International normalized ratio; SD: Standard deviation; * $p$ value was calculated by paired $t$-test. Bias: CoaguChek XS Plus INR - laboratory CA-1500 INR. Mean INR bias: $\Sigma$ (CoaguChek XS Plus INR laboratory CA-1500 INR)/n 


\section{Performance validation}

\section{Precision of the CoaguChek XS Plus system}

The precision of CoaguChek XS was assessed by within-run and between-run CV\%. The results showed a mean of 2.10, an SD of 0.08 , and a CV of $3.60 \%$ for within-run precision and a mean of 2.18 , an SD of 0.07 , and a CV of $3.24 \%$ for between-run precision.

\section{Accuracy of the CoaguChek XS Plus system based on the three ISO criteria}

Figure 1 shows the results of linear regression analysis. For each method, 93 INR values were collected. The correlation between the two methods showed a coefficient of correlation of 0.96 , a slope of 1.05 , and an intercept of -0.14 .

Table 1 presents a summary of the statistical values obtained, including the mean, mean bias, SD, and paired $t$-test, for each subgroup. In this study, the mean INR bias was -0.06 in the INR range of 2.0-4.5 and -0.01 in the entire INR range. Ninety-seven percent of samples with INR $<2$ had a bias of less than \pm 0.5 INR. All of the samples with an INR between 2.0 and 4.5 had an INR bias within \pm 0.3 INR .

Figure 2 shows the bias plot. The bias plot reveals only two outliers in the entire INR range, one with an INR value below 2 and the other with an INR value $>4.5$. These two outliers were identified by the CA-1500 method and the CoaguChek as INR 1.4 and 2.0, respectively, and 5.5 and 7.7, respectively.

According to the results, the criteria of ISO17593:2007 standard were met with the slope, mean bias, and the percentage of the bias in the INR range below 2 INR; only the intercept was slightly outside the range $\pm 0.1 \mathrm{INR}$.

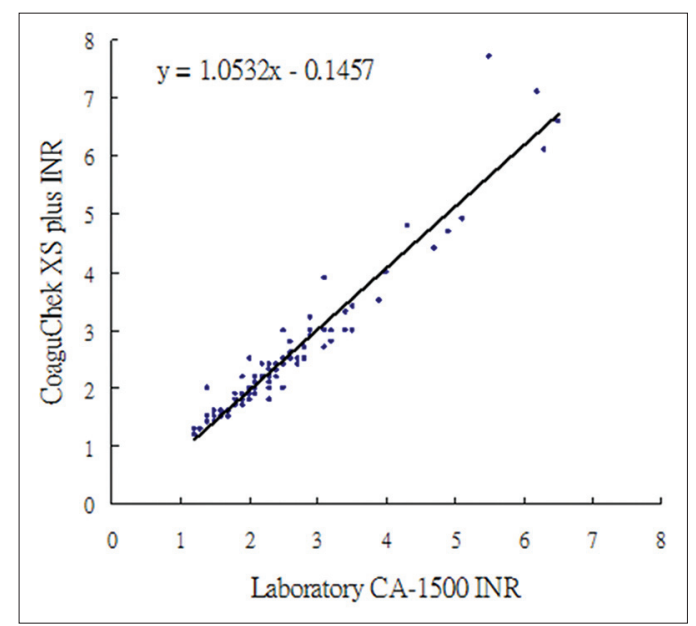

Figure 1: Method comparison by scatter plot with the entire range: $N=93 ; y=1.0532 x-0.1457, r=0.96$. Central laboratory CA-1500: INR mean 2.55; range of 1.2-6.5. CoaguChek XS Plus: INR mean 2.53; range of 1.2-7.7.

\section{Clinical agreement}

The clinical agreement was evaluated by determining whether the INR values of the two methods warranted the same medical prescription. In particular, the clinical agreement was evaluated with respect to whether or not the INR values fell within the same categories. Table 2 shows that $84(90.3 \%)$ samples were categorized in the same subgroup. Three (3.2\%) of the values obtained by CoaguChek XS Plus were falsely higher than those obtained by the CA-1500 method, and six (6.4\%) were falsely lower.

\section{Interference of clinical and genetic factors}

We investigated whether clinical and genetic factors caused the discrepancies in the INR values that resulted in the two outliers on the bias plot. Patients with an INR bias $\geqq 0.5$ in the entire detection range were also investigated. Ten samples from eight patients were selected.

Fisher's exact test indicated a significant difference in the liver function if AST was $>34 \mathrm{u} / \mathrm{L}(3 / 8,37.5 \%$ vs. $3 / 42$, $7.1 \%, p=0.044)$ and ALT was $>36 \mathrm{u} / \mathrm{L}(3 / 8,37.5 \%$ vs. $3 / 42$, $7.1 \%, p=0.044)$. Patients with hypoalbuminemia $(3 / 8,37.5 \%$ vs. $13 / 42,31 \%, p=0.699)$, elevated creatinine $(3 / 8,37.5 \%$ vs.

Table 2: Agreement assessment of CoaguChek XS Plus system and laboratory CA-1500 results in the three INR categories

\begin{tabular}{lccccc}
\hline & \multirow{2}{*}{$\begin{array}{c}\text { INR } \\
\text { range }\end{array}$} & \multicolumn{3}{c}{ Laboratory CA-1500 } & Total \\
\cline { 3 - 5 } & $<2.0$ & $2.0-3.5$ & $>3.5$ & \\
\hline CoaguChek & $<2.0$ & 29 & 5 & - & 34 \\
XS Plus & $2.0-3.5$ & 2 & 45 & 1 & 48 \\
system & $>3.5$ & - & 1 & 10 & 11 \\
& Total & 31 & 51 & 11 & 93 \\
\hline
\end{tabular}

Abbreviation: INR: International normalized ratio

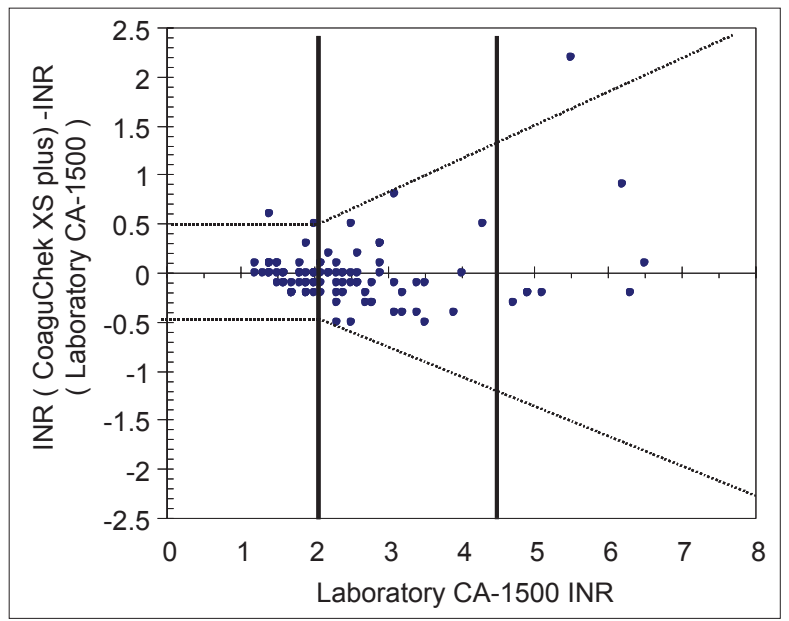

Figure 2: Method comparison by a bias plot of the entire range; stippled lines represent \pm 0.5 INR when is INR $<2$ and $\pm 30 \%$ INR when INR is $\geq 2$, solid vertical lines represent the therapeutic range defined by ISO17593:2007 standard. 
$11 / 42,26.2 \%, p=0.670)$, and anemia $(2 / 8,25.0 \%$ vs. $14 / 42$, $33.3 \%$ ) did not show a significant difference in the precision of the tests and for the various clinical and genetic factors. The genetic factors of VKORC1 (VKORC1-1639 GG 0/8, $0 \%$ vs. $1 / 42,2.4 \%, p=1.0$; AG $1 / 8,12.5 \%$ vs. $5 / 42,11.9 \%$, $p=1.0$; and AA $7 / 8,87.5 \%$ vs. $36 / 42,85.7 \%, p=1.0)$ and CYP2C9 (1*3*1/8, $12.5 \%$ vs. $1 / 42,2.4 \%, p=0.297$ and $1 * 1 * 7 / 8,87.5 \%$ vs. $41 / 42,97.6 \%)$ all displayed no significant difference in the precision of the tests and for the various genetic factors [Table 3].

\section{DISCUSSION}

To our knowledge, this study is the first to evaluate the performance of the CoaguChek XS Plus system in Taiwan that considers the effects of clinical and genetic factors. We evaluated the performance of the CoaguChek XS Plus system using the three requirements of the ISO 17593:2007 standard. In the linear regression analysis, the coefficient of correlation $(r)$ of 0.96 and a slope that fell within the range 0.95-1.05 fulfilled the ISO requirements. However, the intercept at 0.14 was slightly outside the range of \pm 0.1 INR. The intercept outside the allowable range may have been because our sample size was small. According to the ISO 17593:2007 standard, a sample size of 200 subjects and a correct selection of the sample distribution are required. The sample size limited the data performance and increased data variation.

The mean bias in the INR range of 2.0-4.5 was -0.06 INR, which fulfilled the criterion of being within \pm 0.3 INR. Table 1 shows that the mean and SD of the INR values between the two methods were extremely close to each other when the INR was below 4.5. When the INR was above 4.5,

Table 3: Interference of CoaguChek XS Plus system by clinical and genetic factors

\begin{tabular}{lccc}
\hline & \multicolumn{2}{c}{$\begin{array}{c}\text { Positive patient } \\
\text { number }(\%)\end{array}$} & $p$ value* \\
\cline { 2 - 3 } & $\begin{array}{c}\text { Bias } 0.5 \\
n=8\end{array}$ & $\begin{array}{c}\text { Bias }<0.5 \\
n=42\end{array}$ & \\
\hline VKORC1-1639 GA or GG type & $1(12.5)$ & $6(14.3)$ & 1.000 \\
CYP2C9 1*3* AC type & $1(12.5)$ & $1(2.4)$ & 0.297 \\
AST>34 U/L & $3(37.5)$ & $3(7.1)$ & 0.044 \\
ALT>36 U/L & $3(37.5)$ & $3(7.1)$ & 0.044 \\
Albumin <4.0 g/dl & $3(37.5)$ & $13(31.0)$ & 0.699 \\
Elevated Cr** & $3(37.5)$ & $11(26.2)$ & 0.670 \\
Anemia+ & $2(25.0)$ & $14(33.3)$ & 1.000 \\
Hyper- or hypothyroidism & $3(37.5)$ & $4(9.6)$ & 0.071 \\
\hline Abbrevin & & &
\end{tabular}

Abbreviations: VKORC1-1639 GA or GG type: Vitamin K epoxide reductase complex 1 polymorphisms GA or GG type; CYP2C9 $1 * 3 *$ AC type: Cytochrome P-450 2C9 AC type; AST: Aspartate aminotransferase; ALT: Alanine aminotransferase; Cr: Creatinine. Elevated $\mathrm{Cr}$ was defined as serum creatinine $>1.27 \mathrm{mg} / \mathrm{dl}$ in men and $>1.03 \mathrm{mg} / \mathrm{dl}$ in women. Anemia was defined as $\mathrm{Hb}<13.5 \mathrm{~g} / \mathrm{dl}$ in men and $<12 \mathrm{~g} / \mathrm{dl}$ in women. * $p$ value was conducted by $F$-test the SD was larger when compared with the other groups. Related studies have demonstrated that when the INR values exceeded the therapeutic range, the bias increased, especially when the INR values were above 4.5. ${ }^{[14,15]}$ The higher SD when the INR was above 4.5 related to both the World Health Organization (WHO) guidelines, which exclude samples with an INR outside the range of 1.5-4.5 for the ISI assignment. ${ }^{[16]}$ Also, ISO 17593:2007 excludes samples with an INR beyond 6.0. Although the standard deviation was high when the INR value was above 4.5 , the paired $t$-test indicated that the two methods were the same $(p>0.05)$ in the entire range and in each subgroup.

From the eight selected patients, 10 sets of INR values were $\geqq 0.5$, those being (CA-1500/CoaguChek XS Plus): (2.5/2.0), (3.5/3.0), (5.5/7.7), (2.0/2.5), (6.2/7.1), (2.3/1.8), (2.5/3.0), (4.3/4.8), (1.4/2.0), and (3.1/3.9). Four of these sets of INR values, (1.4/2.0), (5.5/7.7), (6.2/7.1), and (3.1/3.9), as well as the two outliers in the bias plot showed exceptionally high variation. This variation might have resulted from clini$\mathrm{cal}$ and genetic interferences. Although Plesch et al. found no significant variation between the CoaguChek XS and venous blood, ${ }^{[6]}$ our study showed that patients who had an INR bias $\geqq 0.5$ had elevated AST and ALT levels that both reached the significant impact level.

Previous studies have experienced interference because of technical problems and antiphospholipid antibody. Lisman et al. indicated that the insufficient performance of INR measurements in patients with end-stage liver disease was because the calibration reagent of the international standard index reagent did not come from liver disease patients, but came from normal pooled plasma. ${ }^{[17]}$ Although the patients in this study did not have severe liver disease, our findings of abnormal AST and ALT levels suggest that plasma protein, such as albumin, or other globulins produced by the hepatobiliary system, may interfere with the CoaguChek XS Plus system.

Is CoaguChek XS Plus system is still reliable in patients with abnormal liver function tests? The INR data sets of the three patients with abnormal liver function were (CA-1500/CoaguChek XS Plus): (2.5/2.0), (3.5/3.0, (5.5/7.7), (2.0/2.5), (3.1/3.9), and (2.2/2.4). Although the INR bias is $\geqq 0.5$, the data sets will be classified into the same subgroups of clinical agreement range and also lead to the same dosage. Abnormal liver function is related to higher INR bias, but does not have an influence on dosage at this stage. So, CoaguChek XS Plus system is still reliable in the patients. However, limited by the sample size, these findings require further investigation.

Clinical agreement was used to decide on patients' dosages. In total, nine cases were classified into subgroups. The INR data sets of these nine cases were (1.4/2.0), (1.9/2.2), (2.0/1.9), (2.0/1.9), (2.0/1.8), (2.1/1.9), (2.3/1.8), (3.1/3.9), and (3.9/3.5) from the CA-1500 and CoaguChek XS Plus

Biomed J Vol. 37 No. 6

November - December 2014 
system, respectively. The values with a data bias $\geqq 0.5$, i.e., $(2.3 / 1.8),(3.1,3.9)$, and the outlier $(1.4 / 2.1)$ were included in this group of nine. The other six cases might have occurred because of random variations in the two methods. The inconsistency from both random variation and from interference would result in approximately $10 \%$ of patients receiving the incorrect dosage. This result is superior to the values of $17.8 \%$ and $33 \%$ previously published using differing reference methods. ${ }^{[18,19]}$ The evidence suggests that values obtained from the CoaguChek XS Plus system should regularly be confirmed by the laboratory CA- 1500 method. To avoid incorrect dosages being prescribed, we suggest that the tests be duplicated and the medication dosages be carefully monitored and adjusted.

\section{Conclusions}

This study evaluated the CoaguChek XS Plus system by comparing it with the laboratory CA-1500 method, using Thromborel S reagent. Although the small sample size limited our data reliability, most of the requirements of the ISO 17593:2007 standard were met. Our data showed that approximately $10 \%$ of patients would be prescribed the incorrect dosage, and we therefore suggest duplication of tests when monitoring INR values. Although the genetic factors $V K O R C 1$ and $C Y P 2 C 9$ or the clinical factors hypoalbuminemia and anemia did not reach the significant level, this study find that elevated AST and ALT level have effect on CoaguChek XS system analysis.

\section{REFERENCES}

1. Ansell J, Hirsh J, Dalen J, Bussey H, Anderson D, Poller L, et al. Managing oral anticoagulant therapy. Chest 2001;119:22S-38S.

2. Hirsh J, Dalen J, Anderson DR, Poller L, Bussey H, Ansell J, et al. Oral anticoagulants: Mechanism of action, clinical effectiveness, and optimal therapeutic range. Chest 2001;119:8S-21S.

3. Leichsenring I, Plesch W, Unkrig V, Kitchen S, Kitchen DP, Maclean R, et al. Multicentre ISI assignment and calibration of the INR measuring range of a new point-of-care system designed for home monitoring of oral anticoagulation therapy. Thromb Haemost 2007;97:856-61

4. ISO 17593:2007 standard, $1^{\text {st }}$ edition. Clinical laboratory testing and in vitro medical devices - Requirements for in vitro monitoring systems for self-testing of oral anticoagulant therapy. 2007.

5. Plesch W, Wolf T, Breitenbeck N, Dikkeschei LD, Cervero A, Perez PL, et al. Results of the performance verification of the CoaguChek XS system. Thromb Res 2008;123:381-9.

6. Plesch $\mathrm{W}$ and van den Besselaar AM Validation of the international normalized ratio (INR) in a new point-of-care system designed for home monitoring of oral anticoagulation therapy. Int J Lab Hematol 2009;31:20-5.

7. Bereznicki LR, Jackson SL, Peterson GM, Jeffrey EC, Marsden KA, Jupe DM. Accuracy and clinical utility of the CoaguChek XS portable international normalised ratio monitor in a pilot study of warfarin home-monitoring. J Clin Pathol 2007;60:311-4.

8. Torreiro EG, Fernandez EG, Rodriguez RM, Lopez CV, Nunez JB. Comparative study of accuracy and clinical agreement of the CoaguChek XS portable device versus standard laboratory practice in unexperienced patients. Thromb Haemost 2009;101:969-74.

9. Bereznicki LR, Jackson SL, Peterson GM. Supervised patient self-testing of warfarin therapy using an online system. J Med Internet Res 2013;15:e138.

10. Bauman ME, Black KL, Massicotte MP, Bauman ML, Kuhle S, Howlett-Clyne S, et al. Accuracy of the CoaguChek XS for point-of-care international normalized ratio (INR) measurement in children requiring warfarin. Thromb Haemost 2008;99:1097-103.

11. Bauman ME, Bruce AK, Buchholz H, Kuhle S, Massicotte MP. Accuracy of the CoaguChek XS (R) for POC INR in warfarinised children and adults with ventricular assist devices. Thromb Haemost 2013;110:616-7.

12. Ero MP, Harvey NR, Harbert JL, Janc JW, Chin KH, Barriere SL. Impact of telavancin on prothrombin time and activated partial thromboplastin time as determined using point-of-care coagulometers. J Thromb Thrombolysis 2013.

13. Wen MS, Lee M, Chen JJ, Chuang HP, Lu LS, Chen CH, et al. Prospective study of warfarin dosage requirements based on CYP2C9 and VKORC1 genotypes. Clin Pharmacol Ther 2008;84:83-9.

14. Vacas M, Lafuente PJ, Unanue I, Iriarte JA. Comparative study of two portable systems for oral anticoagulant monitoring. Hematol J $2004 ; 5: 35-8$.

15. Jonsson M, Hillarp A, Svensson P. Comparison between CoaguChek $\mathrm{S}$ - and Owren-type prothrombin time assay for monitoring anticoagulant therapy. Thromb Res 2004;114:83-9.

16. WHO Expert Committee on Biological Standardization. Guidelines for thromboplastins and plasma used to control oral anticoagulant therapy with vitamin K-antagonists. WHO Technical Report Series 1999;889:64-93

17. Lisman T, van Leeuwen Y, Adelmeijer J, Pereboom IT, Haagsma EB, van den Berg AP, et al. Interlaboratory variability in assessment of the model of end-stage liver disease score. Liver Int 2008;28:1344-51.

18. Hur M, Kim H, Park CM, La Gioia A, Choi SG, Choi JH, et al. Comparison of International Normalized Ratio Measurement between CoaguChek XS Plus and STA-R Coagulation Analyzers. Biomed Res Int 2013;2013:213109.

19. Donaldson M, Sullivan J, Norbeck A. Comparison of International Normalized Ratios provided by two point-of-care devices and laboratory-based venipuncture in a pharmacist-managed anticoagulation clinic. Am J Health Syst Pharm 2010;67: 1616-22. 